\title{
Efecto del aCeite esencial de Cymbopogon Citratus (DC.) StAPF "HIERBA LUISA" EN LOS NIVELES DE ANSIEDAD DE ESTUDIANTES \\ DE EDUCACIÓN SECUNDARIA
}

\section{EFFect of the essential oil of Cymbopogon Citratus (DC.) STAPF "HIERBA LUISA" ON ANXIETY LEVELS OF HIGH SCHOOL STUDENTS}

\author{
Marilú Roxana Soto Vásquez* \\ Paúl Alan Arkin Alvarado García** \\ Luis Enrique Rosales Cerquin*** \\ José Cerna Castillo****
}

\section{RESUMEN}

$\mathrm{E}^{\mathrm{l}}$ objetivo de la investigación fue evaluar el efecto del aceite esencial de Cymbopogon citratus (DC.) Stapf "hierba luisa" en los niveles de ansiedad de estudiantes del quinto grado de educación secundaria de una institución educativa pública. Se realizó un estudio cuasiexperimental, pretest y postest, con una muestra de 54 alumnos, divididos en un grupo experimental tratado con aromaterapia del aceite esencial estudiado, vía inhalatoria, y un grupo control, al cual no se le aplicó ningún estímulo. Los niveles de ansiedad fueron evaluados mediante el inventario de ansiedad estado y ansiedad rasgo STAI. Los niveles de ansiedad disminuyeron después de la intervención, encontrándose en los resultados significancia estadística $(\mathrm{p}<0,005)$. Las puntuaciones $\mathrm{D}$ de Cohen fueron superiores a 1,0 indicando cambios significativos en la variable ansiedad. Además se obtuvo un porcentaje de cambio de $25,01 \%$ para ansiedad estado y 20,34\% para ansiedad rasgo. Todo esto indica que la aromaterapia a base del aceite esencial de hierba luisa puede ser considerada una alternativa promisoria para disminuir los niveles de ansiedad.

Palabras clave: aceites esenciales, ansiedad, aromaterapia, hierba luisa

\footnotetext{
* Docente de la Facultad de Farmacia y Bioquímica. Universidad Nacional de Trujillo. Correo electrónico:msoto@unitru.edu.pe

** Licenciado en Psicología. Universidad Señor de Sipán. Correo electrónico: agarciap@crece.uss.edu.pe

*** Médico cirujano, Hospital de Apoyo Chepén.

**** Licenciado en Educación. IE Generalísimo José de San Martín.
} 


\section{ABSTRACT}

The aim of this research was to evaluate the effect of the essential oil of Cymbopogon citratus (DC.) Stapf "lemongrass" on the anxiety levels of fifth grade high school students of a public educational institution. A quasi-experimental, pretest and posttest study was carried out with a sample of 54 students, divided into an experimental group treated with aromatherapy of the essential oil studied, per inhalation, and a control group to which no stimulus was applied. Anxiety levels were assessed by State-Trait Anxiety Inventory (STAI). Anxiety levels decreased after the intervention, with statistical significance $(\mathrm{p}<0.005)$. Cohen's D scores were over to 1 , indicating significant changes in the anxiety variable. In addition, percentages of change of $25.01 \%$ for state anxiety and $20.34 \%$ for trait anxiety were obtained. All this indicates that aromatherapy based on the essential oil of lemongrass can be considered a promising alternative to decrease anxiety levels.

Keywords: lemongrass, essential oils, aromatherapy, anxiety.

\section{INTRODUCCIÓN}

La ansiedad forma parte de la vida de las personas, desde edades tempranas, sea en la infancia o en la adolescencia. En sí, las reacciones de ansiedad y miedo han jugado un papel significativo en la evolución de la especie humana, como mecanismos de defensa frente a los diferentes peligros ambientales, su finalidad última es salvaguardar al individuo de las posibles amenazas. No obstante la ansiedad se vuelve patológica cuando se desencadena sin que exista alguna circunstancia que la justifique, o existiendo esta circunstancia, su intensidad y frecuencia son desproporcionadas ${ }^{1}$.

Los problemas de ansiedad son los más prevalentes en la población general, en especial en la población adolescente ${ }^{2}$. En el ámbito estudiantil, el adolescente está expuesto a diferentes situaciones que pueden valorarse como eventos amenazantes, sean los exámenes, la violencia entre pares, entre otros ${ }^{3}$. Estos pueden generar en el individuo sentimientos de incompetencia, desmotivación, baja autoestima, preocupaciones constantes y síntomas depresivos; lo que puede influenciar significativamente en el rendimiento académico del alumno y provocar deserción escolar, inhibición del potencial académico del alumno, o dificultades para avanzar en una futura carrera técnica o universitaria ${ }^{4,5}$.

Actualmente, la medicina alternativa y complementaria (MAC) tiene mayor aceptación por parte de los profesionales de la salud y del público general que busca nuevas alternativas de tratamiento ${ }^{6-8}$. La MAC se agrupa en las siguientes categorías: los productos naturales, la medicina de la mente y el cuerpo, las prácticas de manipulación basadas en el cuerpo y otras prácticas de medicina alternativa y complementaria9. En la primera categoría se encuentra el uso de productos naturales, el cual es muy amplio, incluyendo en su mayor parte el uso de las plantas medicinales, cuya aplicación terapéutica se denomina fitoterapia. Algunas plantas medicinales contienen unos metabolitos secundarios denominados aceites esenciales, cuya aplicación medicinal ha dado paso a la aromaterapia. Estos aceites esenciales pueden aplicarse a través de la inhalación, masaje, compresas y baños ${ }^{10}$. 
Los aceites esenciales han sido estudiados ampliamente, encontrándose diversas propiedades biológicas como efectos antinociceptivos, anticancerígenos, antivirales, antimicrobianos, antiinflamatorios, anticonvulsivantes, además de antidepresivos y ansiolíti$\cos ^{11}$.

Cymbopogon citratus (DC.) Stapf "hierba luisa" es una yerba perenne perteneciente a la familia Poaceae, que logra alcanzar hasta 2 metros de alto, con hojas aromáticas entre 30 y 100 centímetros, ampliamente distribuida y usada alrededor del mundo en forma de decocción e infusión. Es tradicionalmente usada para los cólicos y otras dolencias estomacales; así como para aliviar el estrés, los resfriados, fiebre, calmar el dolor y hasta para la artritis ${ }^{12}$. Por su alto contenido en vitamina C, es también utilizada ampliamente como a antioxidante, antibacteriano, y antiinflamatorio; así como para el tratamiento de la diabetes, malaria y ansiedad ${ }^{13-15}$.

Bajo este contexto, el objetivo del presente trabajo fue evaluar el efecto del aceite esencial de Cymbopogon citratus (DC.) Stapf en los niveles de ansiedad de estudiantes del quinto grado de educación secundaria de una institución educativa pública de la provincia de Trujillo.

\section{MÉTODOS}

\section{Material vegetal}

Se recolectaron $2 \mathrm{~kg}$ de hojas de Cymbopogon citratus (DC.) Stapf "hierba luisa" de la localidad de Otuzco, región La Libertad. Un ejemplar completo de la especie vegetal fue identificado y depositado en el Herbarium Truxillense (HUT) de la Universidad Nacional de Trujillo.

\section{Extracción del aceite esencial}

Se utilizó el método de destilación por arrastre de vapor de agua. El destilado se separó tomando en cuenta sus propiedades de inmisibilidad y diferencia de densidades entre el agua y el aceite esencial utilizando una pera de decantación de vidrio. Se deshidrataron las impurezas de agua del aceite esencial haciendo uso de $\mathrm{Na}_{2} \mathrm{SO}_{4}$ anhidro. Luego se filtró y se colocó el aceite esencial en frascos de vidrio de color ámbar. Posteriormente, fueron almacenados en una refrigeradora de laboratorio, a una temperatura de $4{ }^{\circ} \mathrm{C}$ hasta su posterior utilización ${ }^{16}$.

\section{Diseño}

Se realizó un estudio cuasiexperimental, pretest y postest, con dos grupos paralelos, constituidos por las secciones A y B del quinto grado de educación secundaria de una institución educativa pública de la provincia de Trujillo. La muestra estuvo constituida por 54 alumnos, la sección A conformó el grupo control (GC) con 25 alumnos; y la sección B con 29 alumnos, constituyó el grupo experimental (GE). 


\section{Instrumentos de evaluación}

Para la medida de los niveles de ansiedad, se aplicó el cuestionario STAI de Spielberg, Gorsuch, y Lushene, el cual consta de 2 partes, cada una compuesta por 20 preguntas. La primera parte evalúa la ansiedad estado y la segunda la ansiedad rasgo. Las preguntas de ansiedad estado, se evalúan en una escala de 0 a 3 puntos, siendo nada (0), algo (1), bastante (2) y mucho (3). Las puntuaciones de las preguntas de ansiedad estado son nada (0), a veces (1), a menudo (2) y casi siempre (3). La suma de las puntuaciones de cada pregunta varían desde un mínimo de 0 puntos hasta un máximo de 60 puntos, correspondiendo una mayor puntuación a una mayor nivel de ansiedad detectado ${ }^{10}$. Los valores de validez y confiabilidad para la población local fueron determinados en un estudio previo, obteniéndose valores entre 0.24 y 0.39 para las escalas ansiedad rasgo y ansiedad estado; además de una confiabilidad con coeficientes de Spearman Brown de 0.90 y 0.92 para las escalas de ansiedad rasgo y estado respetivamente ${ }^{17}$.

\section{Procedimiento}

En primer lugar se gestionaron los permisos pertinentes y se realizó una charla a los padres de familia de ambas secciones sobre el beneficio de los aceites esenciales; así como la inocuidad del aceite esencial de hierba luisa. Luego del asentimiento de los padres, estos firmaron un consentimiento informado para poder aplicar los estímulos experimentales a sus respectivos hijos.

Durante un periodo 15 días, se aplicaron los siguientes estímulos, al grupo control: no se le aplicó ningún estímulo, y al grupo experimental: administración del aceite esencial de Cymbopogon citratus vía inhalatoria, por dispersión en la atmosfera, a través de 8 difusores ambientales colocados, en cada esquina del aula y al medio. Las ventanas fueron cerradas para evitar la dispersión del aroma. Se validó la dosis necesaria de aceite esencial que saturara la sala por 60 minutos, determinándose una dosis de $0.1 \mathrm{~mL}$., de aceite esencial al 2\%. La evaluación de los niveles de ansiedad se realizó antes de la aplicación del estímulo experimental y finalizado el trabajo. Toda la investigación se llevó a cabo bajo las normas estipuladas en la Declaración de Helsinki.

\section{Análisis estadístico}

Se hallaron las medias y desviaciones típicas de la variable ansiedad del grupo control y del grupo experimental en las fases pretest y postest. Los datos no se ajustaron a la distribución normal por lo que se eligieron estadísticos no paramétricos. Para determinar las diferencias significativas entre las puntuaciones medias del grupo control y cada grupo experimental se empleó la U de Man Whitney para muestras independientes, además para comprobar la existencia de diferencias significativas entre las fases de estudio, se empleó la prueba de Wilcoxon para muestras relacionadas. Asimismo, para determinar la magnitud de cambio experimentado por cada grupo experimental tras la intervención se empleó la prueba de D de Cohen y se hallaron los porcentajes de cambio entre las respectivas puntuaciones pretest y postest. Todos los análisis estadísticos se realizaron mediante el paquete estadístico IBM SPSS versión 23.0 


\section{RESULTADOS}

Tabla 1. Diferencias pretest y postest entre el grupo control y grupo experimental

\begin{tabular}{lccccc}
\hline \multirow{2}{*}{ Grupos } & \multicolumn{2}{c}{ Pretest } & \multicolumn{2}{c}{ Postest } & \multirow{2}{*}{$\mathrm{p}^{\mathrm{b}}$} \\
\cline { 2 - 4 } & $\mathrm{M}$ & $\mathrm{DT}$ & $\mathrm{M}$ & $\mathrm{DT}$ & \\
\hline GC & & & & & \\
Ansiedad estado & 33,44 & $\pm 4,07$ & 33,68 & $\pm 3,94$ & 0,180 \\
Ansiedad rasgo & 26,84 & $\pm 4,85$ & 26,96 & $\pm 4,50$ & 0,606 \\
GE & & & & & \\
Ansiedad estado & 33,94 & $\pm 4,02$ & 25,45 & $\pm 4,25$ & $0,000^{*}$ \\
p & 0,581 & & $0,000^{*}$ & & \\
Ansiedad rasgo & 26,03 & $\pm 4,87$ & 20,34 & $\pm 4,32$ & $0,000^{*}$ \\
$\mathrm{p}^{\mathrm{a}}$ & 0,564 & & $0,000^{*}$ & & \\
\hline
\end{tabular}

a Valor de p calculado mediante la prueba de U de Man Whitney para muestras independientes

b Valor de p calculado mediante la prueba de Wilcoxon para muestras relacionadas * $\mathrm{p}<0,005$

Tabla 2. D de Cohen y porcentaje de cambio Postest en el grupo experimental

\begin{tabular}{ccl}
\hline GE & D Postest & \% Postest \\
\hline Ansiedad estado & 2 & $-25,01 \%$ \\
Ansiedad rasgo & 1,5 & $-20,34 \%$ \\
\hline
\end{tabular}

\section{DISCUSIÓN}

En la tabla 1 se pueden observar como los niveles de ansiedad estado y ansiedad rasgo de los alumnos pertenecientes al grupo experimental, disminuyeron después de la intervención con aromaterapia en base del aceite esencial de Cymbopogon citratus; mientras que los niveles de ansiedad de los alumnos pertenecientes al grupo control mantuvieron similar medida tanto en el pre y postest. Asimismo, al comparar las puntuaciones postest del grupo experimental con el grupo control, se observan diferencias altamente significativas sea para ansiedad estado y para ansiedad rasgo, respectivamente ( $\mathrm{p}<0,005)$. También se hallaron diferencias altamente significativas $(\mathrm{p}<0,005)$ entre las fases de estudio del grupo experimental.

La infusión de hierba luisa ha sido usada por algunos sistemas de medicina tradicional para calmar los nervios ${ }^{18}$. Incluso en México es usada como sedativa ${ }^{19}$. En este sentido, se ha comprobado en modelos experimentales con ratones, que el aceite esencial de esta especie vegetal es efectivo contra la ansiedad y la epilepsia ${ }^{20}$. Los aceites esenciales están constituidos por una mezcla de diferentes compuestos. En el caso del aceite esencial de hierba luisa, tiene como principales componentes una mezcla de cis-trans isómeros como el neral y geranial, seguidos del mirceno y geraniol. Sin embargo, diferentes estudios 
demuestran que estos compuestos por sí solos no poseen actividad ansiolítica, lo que conduce a determinar que los compuestos constituyentes de este aceite esencial poseen propiedades sinérgicas ${ }^{21}$. Asimismo, se ha comprobado los efectos ansiolíticos de otros aceites esenciales, como el de Lavandula officinalis ${ }^{22}$. Asimismo, el aceite esencial de lavanda también ha demostrado ser efectivo en la disminución del estrés y la ansiedad de los pacientes ante una cirugía y durante los tratamientos dentales ${ }^{23,24}$. En este mismo sentido, aceites esenciales como los de Cymbopogon martinii "palmarosa", Ocimum basilicum "albahaca", Pelargonium graveolens "geranio" y Valeriana officinalis "valeriana" también han demostrado ser efectivos en problemas de ansiedad ${ }^{25,26}$.

Asimismo, en la tabla 2, se observan las puntuaciones obtenidas en la prueba de D de Cohen y porcentaje de cambio, donde las puntuaciones D de Cohen son superiores a 1, lo que indica presencia de cambios muy importantes. Además, en relación a los porcentajes de cambio, la variable ansiedad estado presentó relativamente un porcentaje de cambio mayor con $25,01 \%$ en comparación con la variable ansiedad rasgo que presento una disminución de la ansiedad del 20,34\%.

Las puntuaciones anteriores reflejan un efecto del aceite esencial de Cymbopogon citratus sobre los niveles de ansiedad estado y rasgo, indicando que las intervenciones con aromaterapia pueden ser tomadas en cuenta en programas de alivio de la ansiedad en las instituciones educativas. En este sentido, se ha discutido mucho sobre el mecanismo de acción de la aromaterapia; sin embargo este no ha está claramente identificado. Se presume que los aromas tiene un efecto en las células olfativas modulando el sistema límbico, por lo que dependiendo del tipo de aroma, se pueden activar diferentes neurotransmisores y tener diferentes efectos, pudiendo estos modular los estados de ánimo ${ }^{27}$. En relación con las propiedades ansiolíticas de algunos aceites esenciales, se ha demostrado que son algunos componentes terpenicos como el linalol pueden activar el sistema parasimpático produciendo un efecto sedante parecido al de las benzodiacepinas ${ }^{28}$. Sin embargo en el caso de la presente especie vegetal, linalol no es uno de sus componentes mayoritarios, quedando todavía determinar si alguno de estos o la mezcla en su conjunto de todos estos generan el efecto ansiolítico. Una de las limitaciones de la presente investigación es el pequeño tamaño de muestra; sin embargo estos hallazgos siguen confirmando que la utilización de la aromaterapia, pueden ser alternativas para ser tomada en cuenta en la disminución de la ansiedad de adolescentes. No obstante; se necesitan estudios más profundos para determinar mecanismos de acción y los efectos a largo plazo de este tipo de intervenciones.

\section{CONCLUSIÓN}

- La aromaterapia basada en el aceite esencial de Cymbopogon citratus (DC) Stapf "hierba luisa" puede ser considerada una alternativa promisoria para disminuir los niveles de ansiedad en adolescentes. 


\section{REFERENCIAS BIBLIOGRÁFICAS}

1. Sanz, M. Características clínicas de los trastornos de ansiedad. Rev. Pediatr. Aten. Primaria. 2001; 3(10): 61-71.

2. Sneider, BH. Child Psichopathology from infancy to adolescence. New York. Cambridge University Press. 2014.

3. Trautmann A. Maltrato entre pares o "bullying”. Una visión actual. Rev. Chil. Pediatr. 2008; 79(1): 13-20.

4. Bauselas, E. Ansiedad ante los exámenes: evaluación e intervención psicopedagógica. Educere. 2005; 9(31): 553-557.

5. Álvarez, J., Aguilar J., Lorenzo, J. La ansiedad ante los exámenes en estudiantes universitarios: Relaciones con variables personales y académicas. Rev. Electron. Investig. Psicoeduc. Psigopedag. 2012; 10(26): 333-354.

6. Noales-Gaete, J. Medicina alternativa y complementaria. Rev. Chil. Neuro-Psiquiat. 2004; 42(4): 243-250.

7. Lujaj, E., Lizárraga, Z., Mayor, A., Medrano, K., Medina, H., Goicochea, S. El servicio de Medicina complementaria de EsSalud, una alternativa en el sistema de salud peruano. [carta al editor] Rev. Med. Hered. 2014; 25:105-106.

8. Villar, M., Ballinas, Y., Soto, J., Medina, N. Conocimiento, aceptación y uso de la medicina tradicional, alternativa y/o complementaria por médicos del Seguro Social de Salud. Rev. Peruana. Med. Int. 2016; 1(1): 13-18.

9. National Center For Complementary And Integrative Health. Complementary, Alternative, or Integrative Health: What's In a Name? 2016 [Citado 5 de mayo de 2016] Disponible en: https://nccih.nih.gov/health/integrative-health\#types

10. Kutlu Ak, Yilmaz E, Çeçen D. Effects Of Aroma Inhalation On Examination Anxiety. Teach. Learn. Nurs. 2008; 3(4): 125-130.

11. ALI, B., AL-Wabel, NA., Shams, S., Ahamad, A., Khan, SA., Anwar, F. Essential oils used in aromatherapy: A systemic review. Asian. Pac. J. Trop. Biomed. 2015; 5(8): 601-611.

12. Ekpenyong, CE., AKPAN, E., Nyoh, A. Ethnopharmacology, phytochemistry, and biological activities of Cymbopogon citratus (DC.) Stapf extracts. Chin. J. Nat. Med. 2015; 13(5): 321-337.

13. WAannissorn, B., Jarikasem, S., Siriwangchai, T., Thubthimthed, S. Antibacterial properties of essential oil from Thai medicinal plants. Fitoterapia. 2005; 76(2): 233-236.

14. Costa, G., Grangeia, H., Figueirinha, A., Figueiredo, IV., Batista, MT. Influence of harvest date and material quality on polyphenolic content and antioxidant activity of Cymbopogon citratus infusion. Ind. Crops. Prod. 2016; 83(2016): 738-745.

15. Tchoumbougnang, F., Zollo, PH., Dagne, E., Mekonnen, Y. In vivo antimalarial activity of essential oils from Cymbopogon citratus and Ocimum gratissimum on mice infected with Plasmodium Berghei. Plant. Med. 2005; 71(1): 20-23. 
16. Miranda, M. Método de Análisis de Drogas y extractos. La Habana. Universidad de la Habana. 2002

17. Soto-Vásquez, MR., Alvarado-García, PAA. Aromaterapia a base de aceite esencial de "satureja brevicalyx" "inka muña" y meditación mindfulness en el tratamiento de la ansiedad. Med. Nat. 2016; 10(1):47-52.

18. Carlini, EA., Contar, JDP., Silva-Filho, AR., Silveira-Filho, NG., Frochtengarten, ML., BUENO, OFA. Pharmacology of lemongrass (Cymbopogon citratus STAPF). I. Effects of teas prepared from the leaves on laboratory animals. J. Ethnopharmacol. 1986; 17(1):37-64.

19. Tortoriello, J., Romero, O. Plants used by Mexican traditional medicine with presumable sedative properties: an ethnobotanical approach. Arch. Med. Res. 1992; 23(3): 111-116.

20. Costa, CRA., Gargano, AC., Costa, M. Anxiolytic-like effect of the essential oil from Cymbopogon citratus in experimental procedures in mice. Eur. Neuropsychopharmaco. 2006; 16(4): S475S1475.

21. Costa, CA., Kohn, DO., De Lima, VM., Gargano, AC., Flório, JC., Costa M. The GABAergic system contributes to the anxiolytic-like effect of essential oil from Cymbopogon citratus (lemongrass). J. Ethnopharmacol. 2011; 137(1):828-36.

22. Avelo, L., Fernández, P., Faúndez, B., Zagal, A., Gordon, J., Valenzuela, B. Fitoaromaterapia como complemento para mejorar la salud laboral. Rev. Cubana. Plant. Med. 2011; 16(3):279295.

23. Braden, R., Reichow, S., Halm, MA. The use of the essential oil lavandin to reduce preoperative anxiety in surgical patients. J. Perianesth. Nurs. 2009; 24(6):348-355.

24. Kritsidima, M., Newton, T., Asimakopoulou, K. The effects of lavender scent on dental patient anxiety levels: a cluster randomized-controlled trial. Community. Dent. Oral. Epidemiol. 2010; 38(1): 83-87.

25. Buckle, J. Literature review: should nursing take aromatherapy more seriously? Br. J. Nurs. 2007; 16(2): 116-120.

26. Ali, B., AL-Wabel, NA., Shams, S., Ahamad, A., Khan, SA., Anwar, F. Essential oils used in aromatherapy: A systemic review. Asian. Pac. J. Trop. Biomed. 2015; 5(8): 601-611.

27. KYLE, G. Evaluating the effectiveness of aromatherapy in reducing levels of anxiety in palliative care patients: results of a pilot study. Complement. Ther. Clin. Pract. 2006; 12(2):148-55.

28. Fayazi, S., Babashahi, M., Rezaei, M. The effect of inhalation aromatherapy on anxiety level of patients in preoperative period. Iran. J. Nurs. Midwifery. Res. 2011; 16(4): 278-283. 